\title{
Fomento à pesquisa em alimentação e nutrição pelo Ministério da Saúde no Brasil, entre 2004 e 20081.
}

\author{
Financing research in food and nutrition by the \\ Ministry of Health, in Brazil, between 2004 and 2008.
}

\section{Fomento a la investigación en alimentación y nutrición por el Ministerio de Salud, en el Brasil, de 2004 a 2008.}

\author{
Cássia Pereira das CHAGAS ${ }^{2}$ \\ Denise Oliveira e SILVA ${ }^{3}$ \\ Antonia Angulo-TUESTA ${ }^{4}$
}

\begin{abstract}
RESUMO: O artigo apresenta análise dos investimentos à pesquisa em alimentação e nutrição realizado pelo Departamento de Ciência e Tecnologia do Ministério da Saúde do Brasil (Decit/ MS), no período de 2004-2008. Tratou-se de estudo analítico com a utilização da base de dados PesquisaSaude do Decit/MS. Os resultados demonstraram a necessidade de aumentar o volume de recursos priorizando as regiões Norte, Nordeste e Centro-Oeste e financiar pesquisas em alimentação e nutrição de forma contínua para o fortalecimento das ações estratégicas do campo.

Palavras-chave: Financiamento da Pesquisa, Fontes de Financiamento de Pesquisa, Política Pública.
\end{abstract}

ABSTRACT: This article analyses the research investments in food and nutrition conducted by the Department of Science and Technology of the Ministry of Health (Decit/MS) in the 2004-2008 period. This is an analytical study using data source PesquisaSaude Decit / MS. The results shows the need to increase the amount of resources giving priority to the North, Northeast and Midwest and fund research in food and nutrition for the strengthening of the strategic actions of the field.

Keywords: Research Financing, Support of Research, public policy.

RESUMEN: El artículo presenta análisis de la inversión en investigación sobre alimentación y

1 Este artigo foi elaborado a partir de resultados publicados na dissertação de Mestrado "Análise do fomento à agenda de prioridades de pesquisa em saúde relacionadas ao tema alimentação e nutrição no Brasil entre 2004 e 2008 ”, defendida no Programa de Pós-Graduação em Saúde Pública da Escola Nacional de Saúde Pública Sergio Arouca. Brasília, 2011..

2 Economista, Mestre em Saúde Pública pela Escola Nacional de Saúde Pública Sergio Arouca, Pesquisadora colaboradora. FIOCRUZ. Brasília - DF. Brasil - E-mail: cassia.esaf@gmail.com..

3 Pós-doutorado em Antropologia da Alimentação pela Ecole des Hautes Etudes en Science Sociales de Paris, França. Pesquisadora Titular em Saúde Pública e Coordenadora do Programa de Alimentação Nutrição e Cultura da FIOCRUZ. E-mail: deniluz@,fiocruz.br..

4 Doutora em Ciências da Saúde pela UnB. Docente do Curso de Saúde Coletiva, Faculdade de Ceilândia, Universidade de Brasília (UnB/FCE). E-mail: antoniaangulo@unb.br. . 
nutrición realizado por el Departamento de Ciencia y Tecnología del Ministerio de Salud del Brasil (Decit/MS) en el período de 2004-2008. Se trata de estudio descriptivo usando la base de datos PesquisaSaude del Decit/MS. Los resultados mostraron la necesidad de aumentar los recursos destinados a la investigación en las regiones Norte, Noreste y Centro-Oeste del país y continuar apoyando la investigación en alimentación y nutrición para el fortalecimiento de las acciones estratégicas de este campo.

Palabras clave: Financiación de la Investigación, Fuentes de Financiación de Investigación, Política Pública.

\section{INTRODUÇÃO}

O panorama epidemiológico da situação da alimentação e nutrição brasileira aponta mudanças relevantes no perfil de consumo alimentar. As principais alterações na composição alimentar da população estão relacionadas ao aumento do consumo de gorduras e carboidratos. Os distúrbios nutricionais relacionados ao excesso ou desequilíbrio no consumo alimentar têm determinado tanto a obesidade como o baixo peso.

A Política Nacional de Alimentação e Nutrição (PNAN), desde sua promulgação em 1999, assumiu os aspectos do perfil nutricional num contexto de compreensão epidemiológica em que os fatores relacionados ao consumo alimentar e às condições sociais têm relevância para a determinação das doenças crônicas relacionadas à nutrição com aquelas determinadas pela desigualdade social e pobreza. Uma de suas diretrizes legitimou a necessidade de "ampliação do incentivo para pesquisas a partir das prioridades definidas nesta política" (1) a fim de que os resultados das pesquisas contribuam para a implementação de ações e estratégias para a superação do perfil alimentar e nutricional.

A priorização do governo federal em investimentos em ciência e tecnologia (C\&T) neste campo temático iniciou-se com o Programa Fome Zero, que buscava o enfrentamento da fome e da miséria, em 2003. Simultaneamente, sob a liderança do Departamento de Ciência e Tecnologia (Decit), do Ministério da Saúde (MS) definiu-se prioridades de pesquisa na área de alimentação e nutrição para subsidiar os editais de financiamento de pesquisa nesta área. A subagenda de prioridades de pesquisa em Alimentação e Nutrição contempla os seguintes temas: segurança alimentar; amamentação e alimentação complementar da criança; desnutrição energético-proteica; carências nutricionais por micronutrientes; sobrepeso e obesidade e desenvolvimento e validação de metodologias (2).

O Ministério da Saúde em parceria com o Conselho Nacional de Desenvolvimento Científico e Tecnológico (CNPq) e as Fundações Estaduais de Amparo à Pesquisa (FAPs) e do Distrito Federal, lançou editais em nível nacional e estadual, para o financiamento de pesquisas entre 2004 e 2008 . Nesse período, foram financiados 278 pesquisas em Alimentação e Nutrição com um valor global de R \$ 14,5 milhões. No fomento nacional aprovaram-se 204 projetos em 10 editais, no valor de R\$ 
11,8 milhões; no fomento descentralizado ou Programa Pesquisa para o Sistema Único de Saúde: gestão compartilhada em saúde (PPSUS), 71 projetos em 38 editais, com R\$ 2,5 milhões; e três projetos contratados de forma direta no valor de R $\$ 282 \mathrm{mil}$. Este artigo apresenta os investimentos realizados pelo Decit/MS e parceiros e a contribuição para a implementação da PNAN.

A importância do investimento à pesquisa em alimentação e nutrição foi reafirmada na nova versão da PNAN, publicada em 2011. Na oitava diretriz - pesquisa, inovação e conhecimento em alimentação e nutrição, destaca-se a importância de manter os investimentos em pesquisas de delineamento e avaliação de novas intervenções e de avaliação de programas e ações propostos pela política (3). A atualização da política ocorreu após a realização do Seminário Nacional de Alimentação e Nutrição no SUS: PNAN 10 anos, em maio de 2010, em Brasília, cujo objetivo era avaliar a 'trajetória' da política e propor diretrizes para sua reformulação (4).

As modalidades de fomento nacional e descentralizado são os principais instrumentos utilizados pelo Ministério da Saúde para financiar pesquisas em saúde. O Decit define:

Fomento Nacional provê oportunidades para pesquisadores do país se dedicarem a temas considerados prioritários para a saúde pública. Sua realização acontece por meio do lançamento de editais, em âmbito nacional, com o apoio técnico-administrativo do Conselho Nacional de Desenvolvimento Científico e Tecnológico (CNPq) e da Financiadora de Estudos e Projetos (FINEP) (5).

Fomento Descentralizado: projetos apoiados via Programa Pesquisa para o SUS (PPSUS). Por meio do PPSUS, cada estado da federação lança, a cada dois anos, editais que contemplam diversas áreas temáticas de acordo com as necessidades estaduais. Para a execução desse programa, o Decit tem uma parceria com as fundações de amparo a pesquisa (FAPs) e as secretarias de saúde (SES) e de ciência e tecnologia. O CNPq também desempenha importante papel no desenvolvimento do PPSUS fornecendo apoio político, técnico e administrativo (6).

Este artigo apresenta os resultados da investigação sobre os investimentos nas duas modalidades de pesquisa em alimentação e nutrição realizado pelo Decit/MS, no período de 2004-2008.

\section{METODOLOGIA}

Tratou-se de estudo analítico sobre financiamento concedido pelo Ministério da Saúde e parceiros entre 2004 a 2008, por meio de editais públicos - nacional e estadual. Utilizou-se o banco de dados PesquisaSaúde, coordenado pelo Decit/MS.

No banco de dados as pesquisas em alimentação e nutrição foram identificadas a partir dos filtros: 1) campo subagenda de alimentação e nutrição e 2) campo palavras-chave: alimentação, nutrição, alimentar, micronutrientes, agrotóxicos, alimentos, obesidade, desnutrição, dieta, amamentação, aleitamento, sobrepeso, anemia, consumo e nutricional. 
Foram identificados 221 projetos financiados no primeiro filtro. No segundo filtro foram localizados 400 projetos que continham na descrição do título, do resumo ou dos resultados, pelo menos uma das três palavras-chaves utilizadas simultaneamente na busca. Em seguida, o resumo de cada projeto foi lido. Verificou-se que, das 400 pesquisas, somente 57 tinham relação com o tema da alimentação e nutrição. Assim, o total de projetos financiados foi de 278 por 48 editais públicos.

Foi realizada análise quantitativa das variáveis: 1) unidade federativa (UF) da instituição de vínculo do coordenador proponente; 2) ano de publicação do Edital; 3) nome do edital ao qual o projeto está vinculado ou nome do projeto de contratação direta; 4) subagenda da ANPPS; 5) modalidade de fomento; 6) valor do total de recurso aprovado para o projeto de pesquisa. Os dados foram tabulados com a utilização do programa Microsoft Excel.

O estudo foi aprovado pelo Comitê de Ética em Pesquisa da Escola Nacional de Saúde Pública Sergio Arouca, parecer $n^{\circ}$ 098/10.

\section{RESULTADOS}

Foram encontrados 278 projetos coordenados por 233 pesquisadores, vinculados a 80 instituições de ensino e pesquisa no país.

Entre 2004 e 2008, o Ministério da Saúde e parceiros financiaram 2.747 pesquisas, contemplando as 24 subagendas contidas na ANPPS, com um valor global de R 509 milhões de reais. Desse total de pesquisas financiadas, 1.538 projetos $(56 \%)$ foram custeados com $76,2 \%$ dos recursos destinados ao fomento nacional ( $\mathrm{R} \$ 388$ milhões); 1.123 (40,9\%) projetos com 12,4\% dos recursos do fomento descentralizado ( $\mathrm{R} \$ 63$ milhões); e 86 projetos $(3,1 \%)$ foram contratados diretamente com investimento total de R $\$ 58$ milhões.

Do total de subagendas que compõe a ANPPS, a subagenda de Alimentação e Nutrição encontrouse entre as dez que recebeu maior volume de recursos $(2,2 \%)$ e financiou maior número de projetos $(8 \%)$.

Foram financiados 278 projetos de pesquisas em Alimentação e Nutrição com um valor global de R \$ 14,5 milhões. No fomento nacional, 204 projetos receberam o montante de R \$11,8 milhões; e no fomento descentralizado, 71 projetos receberam R\$ 2,5 milhões. Ademais, três projetos foram contratados de forma direta no valor de R\$ 282 mil. Somente $2 \%$ dos recursos foram investidos em contratação direta. Considerando a sua pouca representatividade se comparada com as outras modalidades, essa modalidade não será abordada nos próximos tópicos. Dessa forma, serão considerados 275 projetos e o valor total de recursos, R 14,2 milhões na apresentação dos resultados.

O fomento nacional destinou maior parcela de recursos, quase cinco vezes mais do que o descentralizado, e financiou $73 \%$ do total de projetos no período estudado. Observa-se, ainda, 
que no fomento descentralizado foram lançados três vezes mais editais que no fomento nacional (Tabela 1).

Tabela 1 - Distribuição dos editais publicados, projetos financiados em alimentação e nutrição e valor total de investimento, segundo modalidade de fomento. Brasil, 2004-2008.

\begin{tabular}{l|r|r|r|r|r|r|}
\hline \multirow{2}{*}{ Tipo de fomento } & \multicolumn{2}{|c|}{ Editais publicados } & \multicolumn{2}{c|}{$\begin{array}{c}\text { Projetos financiados em } \\
\text { Alimentação e Nutrição }\end{array}$} & \multicolumn{2}{c|}{$\begin{array}{c}\text { Recursos concedidos } \\
\text { R\$ (mil) }\end{array}$} \\
\cline { 2 - 7 } & $\mathrm{N}$ & $\%$ & $\mathrm{~N}$ & $\%$ & $\mathrm{R} \$$ & $\%$ \\
\hline Nacional & 10 & 21 & 204 & 73 & 11.791 & 81 \\
Descentralizado & 38 & 79 & 71 & 26 & 2.459 & 17 \\
\hline Total & 48 & 100 & 275 & 100 & 14.251 & 100 \\
\hline
\end{tabular}

Fonte: Brasil, Ministério da Saúde, Departamento de Ciência e Tecnologia. Base de dados Pesquisa Saúde. Acesso em 03/Ago/2010.

Quanto à distribuição de projetos e valor financiado por ano, verifica-se grande concentração de recursos no fomento nacional (90\%) entre 2004 e 2005. Por macrorregião, na Região Sudeste, o fomento nacional financiou projetos nos Estados de São Paulo (48\%), Rio de Janeiro (36\%) e Minhas Gerais (15\%), juntos receberam 99\% do montante. Na Região Nordeste os estados da Bahia e Pernambuco lideram com 38\% e 31\% dos recursos financeiros, respectivamente, com 69\% do total da região. O Estado do Maranhão não teve pesquisas aprovadas nesta modalidade. Na Região Norte, $56 \%$ dos recursos foram para o estado do Amazonas. Os estados do Amapá e Roraima não tiveram projetos financiados. No Centro-Oeste, metade dos recursos foi para o Distrito Federal e, no Sul, o estado do Rio Grande do Sul abarcou 65\% dos recursos da região.

No fomento descentralizado, o Nordeste foi à região com maior concentração de projetos financiados (39\%) e maior volume de recursos recebidos (35\%) (Tabela 2).

Tabela 2 - Distribuição de projetos em alimentação e nutrição financiados e valor total de investimento na modalidade nacional e descentralizado, segundo região. Brasil, 2004-2008.

\begin{tabular}{|c|c|c|c|c|c|c|c|c|}
\hline \multirow{3}{*}{ Região } & \multicolumn{4}{|c|}{ Fomento Nacional } & \multicolumn{4}{|c|}{ Fomento Descentralizado } \\
\hline & \multicolumn{2}{|c|}{$\begin{array}{l}\text { Projetos } \\
\text { financiados }\end{array}$} & \multicolumn{2}{|c|}{$\begin{array}{c}\text { Valor } \\
\mathrm{R} \$(\mathrm{mil})\end{array}$} & \multicolumn{2}{|c|}{$\begin{array}{l}\text { Projetos } \\
\text { financiados }\end{array}$} & \multicolumn{2}{|c|}{$\begin{array}{c}\text { Valor } \\
\mathrm{R} \$(\text { mil) }\end{array}$} \\
\hline & $\mathrm{N}$ & $\%$ & $\mathrm{R} \$$ & $\%$ & $\mathrm{~N}$ & $\%$ & $\mathrm{R} \$$ & $\%$ \\
\hline Sudeste & 83 & 41 & 4.697 & 40 & 11 & 15 & 467 & 19 \\
\hline Nordeste & 48 & 24 & 3.139 & 27 & 28 & 39 & 868 & 35 \\
\hline Sul & 34 & 16 & 1.657 & 13 & 11 & 15 & 300 & 12 \\
\hline Centro-Oeste & 26 & 13 & 1.517 & 13 & 11 & 15 & 289 & 12 \\
\hline Norte & 13 & 6 & 779 & 7 & 10 & 14 & 535 & 22 \\
\hline Brasil & 204 & 100 & 11.791 & 100 & 71 & 100 & 2.459 & 100 \\
\hline
\end{tabular}

Fonte: Brasil, Ministério da Saúde, Departamento de Ciência e Tecnologia. Base de dados Pesquisa Saúde. Acesso 
em 03/Ago/2010.

No fomento nacional, as três regiões (Centro-Oeste, Norte e Nordeste) receberam juntas $47 \%$ do montante total, e no fomento descentralizado $69 \%$ (Tabela 2),

No Estado de São Paulo, há 47 grupos de pesquisa em Nutrição, sendo que 19 estão localizados na Universidade de São Paulo (USP), A tabela 3, no que se refere ao fomento nacional, mostra que a Universidade de São Paulo-USP ocupa o primeiro lugar (13,3\%), entre as dez que mais receberam recursos para o financiamento de projetos, do total de 65 instituições participantes na modalidade fomento nacional.

A Universidade Federal da Bahia ocupou o primeiro lugar (16,5\%) entre as dez instituições que mais receberam recursos do fomento descentralizado, do total de 43 participantes nesta modalidade (Tabela 3).

Tabela 3 - Distribuição de projetos financiados e valor de investimento, segundo 10 maiores instituições executoras em total de recursos na modalidade fomento nacional e descentralizado. Brasil, 2004-2008.

\begin{tabular}{|c|c|c|c|c|c|c|c|c|c|c|}
\hline & \multicolumn{5}{|c|}{ Fomento Nacional } & \multicolumn{5}{|c|}{ Fomento Descentralizado } \\
\hline & & & $\begin{array}{l}\text { jetos } \\
\text { ciados }\end{array}$ & & & & $\begin{array}{r}\mathrm{Pr} \\
\text { fina }\end{array}$ & $\begin{array}{l}\text { jetos } \\
\text { iciados }\end{array}$ & $\mathrm{Va}$ & \\
\hline & $\begin{array}{l}\text { Instituição } \\
\text { executora }\end{array}$ & $\mathrm{N}$ & $\%$ & $\begin{array}{l}\mathrm{R} \$ \\
\text { (mil) }\end{array}$ & $\%$ & $\begin{array}{l}\text { Instituição } \\
\text { executora }\end{array}$ & $\mathrm{N}$ & $\%$ & $\begin{array}{l}\mathrm{R} \$ \\
\text { (mil) }\end{array}$ & $\%$ \\
\hline 1 & $\begin{array}{l}\text { Universidade de } \\
\text { São Paulo }\end{array}$ & 26 & 12,6 & 1.547 & 13,3 & $\begin{array}{l}\text { Universidade } \\
\text { Federal da Bahia }\end{array}$ & 6 & 8,5 & 406 & 16,5 \\
\hline 2 & $\begin{array}{l}\text { Universidade } \\
\text { Federal da Bahia }\end{array}$ & 9 & 4,4 & 835 & 7,1 & $\begin{array}{l}\text { Universidade } \\
\text { Federal do Acre }\end{array}$ & 3 & 4,2 & 200 & 8,1 \\
\hline 3 & $\begin{array}{l}\text { Universidade } \\
\text { Federal do Rio de } \\
\text { Janeiro }\end{array}$ & 11 & 5,4 & 759 & 6,4 & $\begin{array}{l}\text { Universidade } \\
\text { Federal de } \\
\text { Viçosa }\end{array}$ & 3 & 4,2 & 162 & 6,6 \\
\hline 4 & $\begin{array}{l}\text { Universidade } \\
\text { Federal de } \\
\text { Pernambuco }\end{array}$ & 14 & 6,7 & 736 & 6,3 & $\begin{array}{l}\text { Universidade } \\
\text { Federal de Minas } \\
\text { Gerais }\end{array}$ & 3 & 4,2 & 153 & 6,2 \\
\hline 5 & $\begin{array}{l}\text { Universidade de } \\
\text { Brasília }\end{array}$ & 13 & 6,3 & 634 & 5,4 & $\begin{array}{l}\text { Universidade } \\
\text { Federal de } \\
\text { Alagoas }\end{array}$ & 8 & 11,3 & 150 & 6,1 \\
\hline 6 & $\begin{array}{l}\text { Universidade } \\
\text { Federal de Mato } \\
\text { Grosso }\end{array}$ & 3 & 1,5 & 534 & 4,5 & $\begin{array}{l}\text { Instituto } \\
\text { Nacional de } \\
\text { Pesquisa da } \\
\text { Amazônia }\end{array}$ & 2 & 2,8 & 130 & 5,3 \\
\hline 7 & $\begin{array}{l}\text { Universidade do } \\
\text { Estado do Rio de } \\
\text { Janeiro }\end{array}$ & 6 & 2,7 & 408 & 3,6 & $\begin{array}{l}\text { Universidade de } \\
\text { Brasília }\end{array}$ & 2 & 2,8 & 112 & 4,6 \\
\hline 8 & $\begin{array}{l}\text { Universidade } \\
\text { Federal de Pelotas }\end{array}$ & 6 & 3 & 330 & 2,9 & $\begin{array}{l}\text { Universidade } \\
\text { Federal do } \\
\text { Tocantins }\end{array}$ & 2 & 2,8 & 101 & 4,1 \\
\hline
\end{tabular}




\begin{tabular}{|c|c|c|c|c|c|c|c|c|c|c|}
\hline 9 & $\begin{array}{l}\text { Universidade } \\
\text { Federal de Minas } \\
\text { Gerais }\end{array}$ & 4 & 2 & 319 & 2,8 & $\begin{array}{l}\text { Universidade } \\
\text { Regional de } \\
\text { Blumenau }\end{array}$ & 1 & 1,4 & 87 & 3,6 \\
\hline 10 & $\begin{array}{l}\text { Universidade } \\
\text { Federal de Santa } \\
\text { Catarina }\end{array}$ & 7 & 3,2 & 283 & 2,5 & $\begin{array}{l}\text { Universidade } \\
\text { Federal da } \\
\text { Paraíba }\end{array}$ & 2 & 2,8 & 79 & 3,2 \\
\hline & Total & 99 & 47,8 & 6.390 & 54,8 & Total & 32 & 45 & 1.585 & 64,3 \\
\hline
\end{tabular}

Fonte: Brasil, Ministério da Saúde, Departamento de Ciência e Tecnologia. Base de dados Pesquisa Saúde. Acesso em 03/Ago/2010.

\section{DISCUSSÃO}

Um número alto de projetos $(96,1 \%)$ receberam recursos do Decit/MS, levando-se em conta o volume de recursos concentrados em 2004 e 2005 para um número reduzido de editais no fomento nacional. Foram dois editais lançados em parceria com o CNPq e destinados exclusivamente ao tema da Alimentação e Nutrição: Edital MCT/CNPq/MS/CT-Saúde nº. 030/2004 e Edital MCT/ CNPq/MS-SCTIE-DECIT/SAS-DAB nº 051/2005. O investimento não foi contínuo e estável, nota-se uma redução de recursos para o financiamento de pesquisas a partir de 2006.

O elevado número de pesquisadores contemplados explica-se pelo fato da exigência de titulação restringir-se ao título de especialista; e em 2005, a titulação de mestre, nas regiões Centro-Oeste e Norte fomentaram um maior número de pesquisadores pleiteantes ao fomento.

As instituições contempladas com recursos nos estados da federação tem vinculação com programas de pós-graduação em nutrição (7). Em São Paulo, a USP, por ser uma das maiores instituições públicas do estado, destacaram na captação de recursos e, no Rio de Janeiro, a UFRJ teve o mesmo tratamento, em função de deter, ambas instituições de ensino, quatro programas de pós-graduação em Nutrição. No Nordeste, os programas de nutrição das entidades de ensino UFBA (Bahia), UFPE (Pernambuco), UFPB (Paraíba), UFAL (Alagoas) e UFRN (Rio Grande do Norte) também foram as contemplados por editais de fomento.

No fomento descentralizado, o Nordeste foi à região com maior concentração de projetos financiados (39\%) e maior volume de recursos recebidos (35\%) (Tabela 2). Do modo geral os recursos foram concentrados em cinco estados do país, dos 23 contemplados. Minas Gerais abarcou $78 \%$ do total da Região Sudeste (R\$ 365 mil); e o estado da Bahia financiou oito projetos com 52\% do valor total do Nordeste (R $\$ 442$ mil). O Rio Grande do Norte não teve pesquisas selecionadas. No Sul, Santa Catarina investiu 53\% dos recursos da região em quatro projetos, e no Norte, o Acre financiou três projetos com R\$ 200 mil. E, na Região Centro-Oeste, o Distrito Federal com 39\% (do total da região) apoiou apenas dois projetos, enquanto o Mato Grosso, com valor menor (R $\$ 66$ mil), desenvolveu cinco pesquisas. Os dados também podem ser compreendidos sob o aspecto das instituições executoras. 
A respeito da distribuição regional, conforme determinado pelo Artigo $3^{\circ}$ do Decreto $\mathrm{N}^{\circ} 4.143$, de 25/02/2002 (8), os editais publicados deveriam destinar parcela mínima de 30\% do montante para projetos desenvolvidos por pesquisadores vinculados a instituição de ensino superior ou de pesquisa sediadas nas regiões Norte, Nordeste e Centro-Oeste. Essa estratégia é para fomentar a pesquisa nessas regiões menos favorecidas. Os resultados demonstraram que a distribuição de recursos por macrorregiões atendeu a determinação legal. Essa estratégia refletiu a preocupação com a quebra da hegemonia das concentrações espacial e institucional, resultado das grandes desigualdades regionais.

No que se refere ao fomento descentralizado, os editais foram elaborados com base na situação de saúde dos estados com vistas a priorizar os problemas prevalentes na população. Essa modalidade de financiamento estipulou que somente poderia concorrer o pesquisador vinculado a instituições de ensino e pesquisa no estado em que publicou o edital de fomento.

Os editais regionais também têm algumas desvantagens, pois o recurso disponibilizado depende da contrapartida estadual respectiva, que influenciará na parcela a ser concedida pelo Decit. Alguns estados da federação não possuem recursos orçamentários para fazer face a essas contrapartidas exigidas pelo âmbito federal.

\section{CONCLUSÃO}

Espera-se superar os desafios para o fomento nacional e descentralizado no que diz respeito à: (1) continuar a priorização nas regiões Norte, Nordeste e Centro-Oeste em função da transição nutricional; (2) aumentar o volume de recursos com sustentabilidade e regularidade de lançamento de editais; (3) melhorar a governança, principalmente na melhoria dos aspectos gerenciais das Fundações de Amparo à Pesquisa-FAPs.

É imperioso fortalecer a capacidade regional de C\&T com a criação de centros de pesquisa e inovação, capacitação de recursos humanos e o fortalecimento dos profissionais de ensino superior e de cursos de pós-graduação nas regiões mais pobres do país.

A manutenção e regularidade de lançamento de editais de pesquisa contribui com o desenvolvimento de programas e políticas públicas no campo da Alimentação e Nutrição no Brasil.

A governança do fomento descentralizado tem sido afetada pelos problemas políticoinstitucionais das FAPs. Há necessidade de superar desafios e avançar para um modelo de governança que de fato contribua para diminuir as desigualdades regionais com a revisão de integração de ações comuns entre órgãos de C\&T como a CAPES, CNPq e FINEP. 
A promulgação da PNAN teve papel fundamental para o campo, pois introduziu o tema na agenda governamental, investiu em ações e programas, além de pesquisas para o combate aos problemas nutricionais do país. A revisão da política trouxe a possibilidade da inclusão de novos temas de investigação que devem ser priorizados pelo Ministério da Saúde com uma expressão diretiva do campo da nutrição.

Para a PNAN, o financiamento de pesquisas em Alimentação e Nutrição representa um componente fundamental para o seu monitoramento e avaliação, pois os resultados dos projetos poderão fornecer subsídios para sua atualização e aperfeiçoamento, bem como para a elaboração de ações e programas. Ademais, a pesquisa demonstrou a integração de expectativas entre pesquisadores brasileiros do campo de nutrição e os órgãos de governo do MS responsáveis pelo financiamento em C\&T em saúde.

\section{REFERÊNCIAS BIBLIOGRÁFICAS}

1. Ministério da Saúde (BR), Secretaria de Ciência, Tecnologia e Insumos Estratégicos. Agenda Nacional de Prioridades de Pesquisa em Saúde. Brasília: Ministério da Saúde, 2008.

2. Ministério da Saúde. Pesquisa em saúde no Brasil. Revista Saúde Pública. Brasília: 2008. [Acesso em 20 Ago 2010]. Disponível em: http://www.scielosp.org/pdf/rsp/v42n4/itdecit.pdf

3. Ministério da Saúde. Secretaria de Atenção à Saúde. Política Nacional de Alimentação e Nutrição Brasília: Ministério da Saúde, 2012.

4. Ministério da Saúde (BR), Coordenação Geral da Política de Alimentação e Nutrição. Seminário Nacional de Alimentação e Nutrição no SUS - PNAN 10 Anos. Brasília: Ministério da Saúde, 2010.

5. Ministério da Saúde (BR). Secretaria de Ciência, Tecnologia e Insumos Estratégicos. Pesquisa para saúde: contribuições aos 20 anos do SUS. Brasília: Ministério da Saúde, 2008.

6. Ministério da Saúde. Dicionário de dados: informações básicas de utilização do sistema. Brasília: 2010. [Acesso em 03 Ago 2010]. Disponível em: http://pesquisasaude.saude.gov.br/ bdgdecit/dicionario.php

7. Coordenação de Aperfeiçoamento de Pessoal de Nível Superior. Brasília: 2010. [Acesso: 20 dez. 2010]. Disponível em: http://www.capes.gov.br

8. Brasil. Decreto $n^{\circ} 4.143$, de 25 de Fevereiro de 2002. Regulamenta a Lei ${ }^{\circ}$ 10.332, de 19 de dezembro 
de 2001, na parte que institui mecanismo de financiamento para o Programa de Fomento à Pesquisa em Saúde, e dá outras providências. Diário Oficial da República Federativa do Brasil. (26 fev. 2002); Seção 1, p. 1-2.

Artigo apresentado em 15-11-14

Artigo aprovado em 20-06-15 Artigo publicado no sistema em 22-12-15 